\title{
Relationship of Sulcular Sulfide Level to Severity of Periodontal Disease and BANA Test
}

\author{
Manabu Morita* and Hom-Lay Wang ${ }^{\dagger}$
}

Background: Volatile sulfur compounds (VSC), such as hydrogen sulfide and methyl mercaptan, are toxic metabolites produced by periodontal pathogens. Their relationship to periodontal disease severity is not yet fully understood. Hence, the aims of this study were to: 1) examine the relationship between sulcular sulfide (pS) levels and severity of periodontal disease and 2) examine the link between pS level and the BANA (benzoyl-DLarginine-naphthylamide) test.

Methods: Seventy systemically healthy subjects with a mean age of $53.0 \pm 13.8$ years participated. Three sites were selected from each subject based upon radiographic bone loss (RBL): RBL $<2 \mathrm{~mm}$, healthy; RBL $\geq 2$ to $<4$, low to moderate; RBL $\geq 4 \mathrm{~mm}$, severe. Periodontal parameters, probing depth (PD), clinical attachment level (CAL), and bleeding on probing (BOP), were recorded. The pS level was measured using a portable sulfide monitor in a digital score ranging from $0.0\left(<10^{-7} \mathrm{M}\right.$ of S) to $5.0\left(\geq 10^{-2} \mathrm{M}\right.$ of $\left.S\right)$ in increments of 0.5 . The presence of specific bacteria in subgingival plaque was detected using BANA test.

Results: The mean pS level was $0.10 \pm 0.23,0.36 \pm 0.48$, and $1.10 \pm 0.87$ for healthy, low to moderate, and severe disease sites, respectively, and was statistically different $(P<0.001)$. The pS level was positively correlated with the BANA test, and was higher in untreated subjects than maintenance subjects $(P$ $<0.01$ ).

Conclusions: The pS level may be a potential indicator for detecting severity of periodontal disease and identifying bacteria that are capable of hydrolyzing BANA. J Periodontol 2001; 72:74-78.

\section{KEY WORDS}

Gingival crevicular fluid/analysis; BANA test; gingivitis; periodontal diseases/diagnosis; sulfur compounds.

\footnotetext{
* Previously, Department of Preventive Dentistry, Okayama University Dental School, Okayama, Japan; currently, Department of Preventive Dentistry, Hokkaido University Dental School, Sapporo, Japan.

$\dagger$ Department of Periodontics/Prevention/Geriatrics, University of Michigan School of Dentistry, Ann Arbor, MI
}

I oxic metabolites of bacterial origin are implicated as important destructive factors in periodontal disease. Volatile sulfur compounds (VSC), such as hydrogen sulfide, methyl mercaptan, and dimethyl sulfide are toxic metabolites produced by microorganisms in the periodontal environment. ${ }^{1-6} \mathrm{~A}$ few clinical reports have shown positive correlations among sulcular sulfide levels to degree of gingival inflammation ${ }^{7}$ and probing depth. ${ }^{8-10}$ However, the relationship between sulcular sulfide level and the severity of periodontal disease remains unknown.

For determination of VSC in periodontal pockets, gas chromatography has often been used. 7,9,10 However, this measurement device requires some technical expertise and is costly and time consuming. Hence, it is not practical for clinical usage. Recently, a portable monitor that provides real-time chairside information on sulcular sulfide levels (pS levels) has been developed. With this device, it has become possible to measure sulfide levels in periodontal pockets.

In an in vitro study, Persson et al. ${ }^{11}$ demonstrated that Gram-negative anaerobic bacteria taken from periodontal pockets were capable of producing hydrogen sulfide and methyl mercaptan in human serum. This is especially true with 3 putative periodontal pathogens, Treponema denticola, Porphyromonas gingivalis, and Bacteroides forsythus, since they produce copious amounts of hydrogen sulfide and methyl mercaptan. ${ }^{12}$ Since these bacteria can be detected 
through their ability to hydrolyze synthetic peptide benzoyl-DL-arginine-naphthylamide (BANA), the BANA test was developed and has been used as a potential diagnostic tool for periodontal disease. ${ }^{13,14}$ However, the relationship between the presence of these bacteria and sulcular sulfide levels has not yet been clarified.

The purpose of this study was to examine: 1) the correlation between periodontal disease severity and pS levels using the portable sulfide monitor and 2) the link between the pS level and BANA test.

\section{MATERIALS AND METHODS}

\section{Study Population}

A power calculation was performed to determine the sample size required. The estimated standard deviation of the measurement parameters (probing depth, clinical attachment level) was chosen as $0.35 \mathrm{~mm}$. The minimum detecting difference was selected at 0.16 mm. ${ }^{15}$ A minimum number of 67 subjects was required to allow a $95 \%$ chance of detecting a statistically significant difference with $\alpha$ set at 0.05 and the power of the study set at $80 \%$.

After the Institutional Review Board of the University of Michigan approved the study protocol, 70 generally or locally advanced periodontal patients being treated in Graduate Periodontics at the University of Michigan School of Dentistry were selected. Subject inclusion criteria were as follows: 1) adult males or females older than 20 years of age and 2) having 3 representative sites with radiographic evidence of bone loss (RBL): RBL $<2.0 \mathrm{~mm}$, healthy; $\mathrm{RBL} \geq 2$ to $<4.0$ $\mathrm{mm}$, low to moderate; and RBL $\geq 4.0 \mathrm{~mm}$, severe disease sites. ${ }^{16}$ Radiographs $^{\dagger}(70 \mathrm{kVp}, 7 \mathrm{~mA})$ were taken using the parallel technique and Rinn holders. $§$ RBL was expressed as the distance in $\mathrm{mm}$ from the cemento-enamel junction (CEJ) to the alveolar crest. Subjects taking any antibiotics within the last 3 months, having evidence of systemic disease (e.g., diabetes, hepatic or renal failure) or infectious disease (e.g., hepatitis, human immunodeficiency virus, or tuberculosis), or evidence of pregnancy or lactation were excluded from the study.

\section{Examination}

Probing depth (PD) and clinical attachment level (CAL) were recorded utilizing the University of North Carolina probe (read to $1 \mathrm{~mm}$ ). CAL was expressed as a distance $(\mathrm{mm})$ from the CEJ to the base of the periodontal pocket. The measurements were made to the nearest $1 \mathrm{~mm}$. BOP was recorded as present or absent 30 seconds after probing. The intra-examiner agreements of PD and CAL were 0.96 and 0.95 , respectively, using the Pearson correlation test, when 30 periodontal pockets were randomly selected from 2 patients and examined on two separate occasions.

The sulcular sulfide (pS) level was recorded using the portable sulfide monitor." This device consists of an

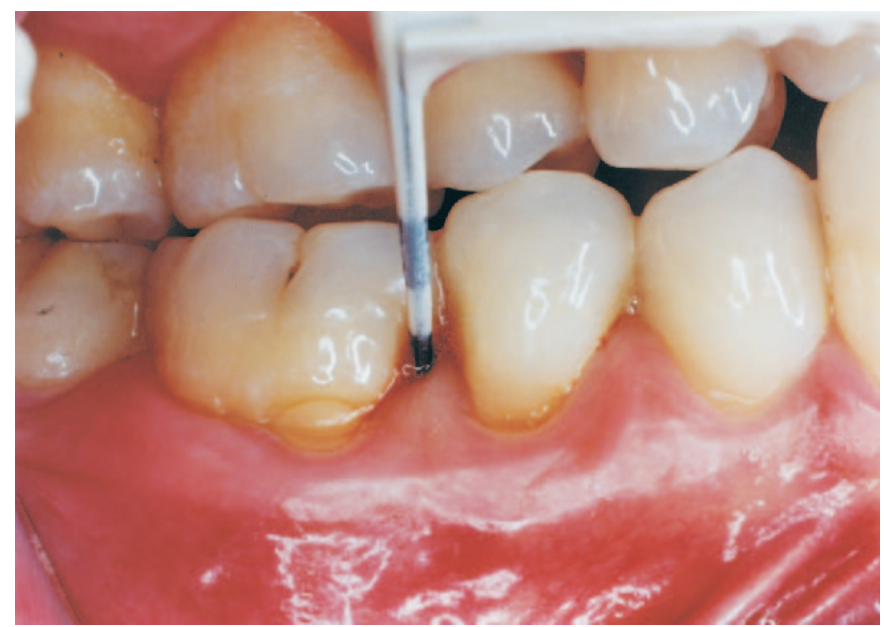

Figure I.

A sulfide sensor tip inserted into a periodontal pocket. The probe tip was inserted into the periodontal crevice in a manner identical to the routine measurement of $P D$.

electric control unit and a disposable sensor tip that combines a standard Michigan "O" style dental probe with a sulfide sensor. The test area was air-dried, and the probe tip was inserted into the periodontal crevice in a manner identical to the routine measurement of PD (Fig. 1). The probe tip with a sulfide sensor responds to the various forms of sulfides expected in periodontally involved pockets. The electronic control unit reports the sulfide level at each site in a digital score ranging from 0.0 (undetectable $\mathrm{pS} ;<10^{-7} \mathrm{M}$ of sulfide) to $5.0\left(\geq 10^{-2} \mathrm{M}\right.$ of sulfide) in increments of 0.5 . This digital score, $\mathrm{pS}$, is defined by $\mathrm{pS}=(7+\log \mathrm{S})$, where $S$ is the molar concentration of sulfide in an "equivalent model sulcus fluid." The intra-examiner pS agreement was 0.95 using the Pearson correlation coefficient test, when examined on two separate occasions.

The commercially available diagnostic test kit based on the hydrolysis of BANA was used to assess the presence of $T$. denticola, $P$. gingivalis, and $B$. forsythus. ${ }^{13}$ After removal of supragingival plaque, subgingival plaque of the 3 representative sites was collected using a sterile curet. The samples were placed on a BANA-impregnated strip along the lower border of a test card. An upper reagent strip containing Evan's black dye was then activated through dampening with distilled water, and the 2 strips were folded over so they contacted one another. After folding, the card was incubated at $35^{\circ} \mathrm{C}$ for 5 minutes. ${ }^{17}$ Results that produced a definite blue and a pale blue were recorded as score 2 (positive) and 1 (weak positive), respec-

\footnotetext{
‡ GX-770, Gendex Corp., Des Plaines, IL.

$\S$ Rinn Corp., Elgin, IL.

| Diamond Probe/Perio 2000, Diamond General Development Corp., Ann Arbor, MI.

If Perioscan, Oral-B, Redwood City, CA.
} 
tively. No reaction was recorded as a 0 (negative).

\section{Statistical Analysis}

The data were expressed as the mean \pm standard deviation. Statistical differences of the mean PD, CAL, pS level, and BANA test were analyzed among 3 sites in RBL groups by a repeated measures analysis of variance (ANOVA). The prevalence of sites with BOP positive were compared in the three bone loss sites by a GEE (generalized estimating equations) approach. The difference of the mean pS and percent of detectable pS ( 0.5 or more) among the sites with different BANA test results were tested by 1 -way ANOVA or chi-square test. The mean value of the $\mathrm{pS}$ and the BANA results of each RBL site were compared between untreated and maintenance patients by 1 -way ANOVA. Calculations were undertaken using a statistical software package."

The pS-positive sites were defined as those with detectable $\mathrm{pS}$ ( $\mathrm{pS} \geq 0.5$ ). The BANA-positive sites were defined as those with either BANA score 1 or 2. Using this criterion, $2 \times 2$ contingency tables were constructed to calculate sensitivity and specificity in relation to BOP. This allowed us to detect the relationship of BOPpositive sites to pS-positive and BANA-positive sites. Sensitivity indicated the probability that BOP-positive sites would yield a positive test result. Specificity indicated the probability that BOP-negative sites would yield a negative test result. ${ }^{18}$

\section{RESULTS}

Subjects consisted of 32 females and 38 males with the mean age of $53.0 \pm 13.8$ years. In total, there were 49 Caucasian, 9 African-American, and 12 Asian subjects. Thirty-seven subjects were undergoing maintenance therapy on a recall schedule of 3 months or longer, while the remaining 33 were untreated subjects.

Table 1 shows the periodontal parameters (PD, CAL, $\mathrm{BOP}$ ), pS level, and BANA test in the 3 representative sites with different severity of bone loss. The mean $\mathrm{pS}$ level was $0.10 \pm 0.23,0.36 \pm 0.48$, and $1.10 \pm 0.87$ for healthy, low to moderate, and severe disease sites, respectively. The highest pS score was 2.5 in the severe sites, which corresponded to a sulfide concentration of between $3 \times 10^{-5}$ and $1 \times 10^{-4} \mathrm{M}$. There were significant differences for all parameters among the 3
Table 2.

Mean pS Level and Percentage of Sites With Detectable pS (pS $\geq 0.5$ ) According to the Results of BANA Test

\begin{tabular}{lcccc}
\hline & \multicolumn{4}{c}{ BANA Test } \\
\cline { 2 - 4 } & Negative & Weak Positive & Positive & $P$ \\
\hline$N$ & 103 & 66 & 41 & \\
Mean \pm SD pS & $0.14 \pm 0.39$ & $0.65 \pm 0.69$ & $1.29 \pm 0.72$ & $*$ \\
$\%$ of detectable $\mathrm{PS}$ & 17.5 & 66.7 & 97.6 & $\dagger$ \\
\hline$* P<0.001$ by 1 1-way ANOVA. & & \\
$\dagger P<0.001$ by chi-square test. & & & \\
\end{tabular}

RBL groups $(P<0.001)$. Significant differences were also observed for all parameters between each 2 sites with different severity of bone loss $(P<0.01)$.

Table 2 shows the relationship between the BANA test result and mean $\mathrm{pS}$ and percentage of detectable pS $(\geq 0.5)$. The mean pS significantly correlated with BANA score (Pearson correlation coefficient $=0.603$, $P<0.001)$. About $98 \%$ of the BANA-positive sites exhibited detectable pS, while only $18 \%$ of the BANAnegative sites had detectable pS.

The mean pS level and BANA score of the sites with different severity of bone loss were compared between the 33 untreated subjects and 37 maintenance sub-

\# SPSS Inc., Chicago, IL. 
Table 3.

\section{Mean Value and Standard Deviation of pS Levels and BANA Test Values in Untreated and Maintenance Subjects}

\begin{tabular}{|c|c|c|c|}
\hline $\begin{array}{c}\text { Radiographic } \\
\text { Bone Loss }\end{array}$ & $\begin{array}{l}\text { Untreated } \\
(n=33)\end{array}$ & $\begin{array}{c}\text { Maintenance } \\
\quad(n=37)\end{array}$ & P \\
\hline \multicolumn{4}{|l|}{ pS level } \\
\hline Healthy & $0.14 \pm 0.29$ & $0.07 \pm 0.17$ & \\
\hline Low to moderate & $0.58 \pm 0.56$ & $0.18 \pm 0.32$ & * \\
\hline Severe & $1.68 \pm 0.7 \mid$ & $0.58 \pm 0.64$ & † \\
\hline \multicolumn{4}{|l|}{ BANA test } \\
\hline Healthy & $0.42 \pm 0.75$ & $0.16 \pm 0.44$ & \\
\hline Low to moderate & $0.94 \pm 0.75$ & $0.49 \pm 0.65$ & $\dagger$ \\
\hline Severe & $1.45 \pm 0.62$ & $0.84 \pm 0.73$ & t \\
\hline
\end{tabular}

jects. As listed in Table 3, the sites with low to moderate and severe bone loss in untreated subjects exhibited significantly higher $\mathrm{pS}$ level and BANA test scores than those in regular maintenance subjects $(P<0.01)$.

The use of $\mathrm{pS}$ level or BANA test to differentiate between BOP-positive sites and BOP-negative sites was tested using the $2 \times 2$ contingency tables (Table 4 ). The sensitivity and specificity of detectable pS related to BOP was $87 \%$ and $83 \%$, respectively. The BANA test showed a sensitivity of $84 \%$ and specificity of $76 \%$.

\section{DISCUSSION}

This study indicates that pS level increased significantly with the severity of periodontal disease (bone loss). The mean pS level was $0.10 \pm 0.23,0.36 \pm 0.48$, and $1.10 \pm 0.87$ for healthy, low to moderate, and severe bone loss sites, respectively. This is in support of the hypothesis proposed by Fine and Mandel. ${ }^{19}$ They suggested that hydrogen sulfide generated by causative bacteria in periodontal disease may be an indicator for initiation or progression of periodontal disease. Furthermore, results obtained from this trial indicated that untreated subjects showed significantly higher $\mathrm{pS}$ than maintenance subjects. This may imply that pS level may be a useful tool for assessing outcome of periodontal therapy.

The present study also reported that pS level significantly correlated with PD and percent of BOP-positive sites. These results are in agreement with previous clinical reports. ${ }^{7-10}$ Solis-Gaffar et al. ${ }^{7}$ measured levels of hydrogen sulfide production in human gingival crevicular fluid (GCF). A significant positive correlation $(r=0.66)$ was obtained between the level of
Table 4.

\section{Diagnostic Ratios of the pS and BANA Test Results Compared With BOP}

\begin{tabular}{|c|c|c|c|c|c|}
\hline \multirow[b]{2}{*}{ pS Level } & \multicolumn{2}{|c|}{$\mathrm{BOP}$} & \multirow[b]{2}{*}{ BANA Test } & \multicolumn{2}{|c|}{$\mathrm{BOP}$} \\
\hline & Positive & Negative & & Positive & Negative \\
\hline Positive* & 82 & 20 & Positive ${ }^{\dagger}$ & 79 & 28 \\
\hline Negative & 12 & 96 & Negative & 15 & 88 \\
\hline \multicolumn{3}{|c|}{ Sensitivity: $82 / 94=87 \%$} & \multicolumn{3}{|c|}{ Sensitivity: $79 / 94=84 \%$} \\
\hline \multicolumn{3}{|c|}{ Specificity: $96 / 116=83 \%$} & \multicolumn{3}{|c|}{ Specificity: $88 / 116=76 \%$} \\
\hline
\end{tabular}

hydrogen sulfide generated in GCF and the GCF volume. Hydrogen sulfide generated in GCF increased with severity of gingival index. ${ }^{20}$ Coil and Tonzetich ${ }^{9}$ analyzed the VSC from gingival crevices of 17 subjects. Crevicular sites that were either deep (PD $\geq 4$ $\mathrm{mm}$ ) or inflamed (BOP positive) exhibited significantly higher total sulfur contents than corresponding shallow (PD $\leq 3 \mathrm{~mm}$ ) or non-inflamed (BOP negative) sites.

The clinical relationship between periodontal disease and VSC is also supported by in vitro studies. ${ }^{2,6}$ The highest pS level score observed in the present study $\left(2.5,3 \times 10^{-5}\right.$ to $1 \times 10^{-4} \mathrm{M}$ of sulfide $)$ is equivalent to 0.96 to $3.2 \mathrm{ppm}$ of sulfide. Experiments have demonstrated that exposure to 2 ppm of hydrogen sulfide and methyl mercaptan reduced total protein production in human gingival fibroblasts by $18 \%$ and $35 \%$, respectively. ${ }^{2}$ Therefore, periodontal pockets have enough VSC to influence the health of the periodontium. Ratcliff and Johnson ${ }^{6}$ reviewed articles describing the possible roles of VSC and speculated that VSC might be actively involved in the pathogenesis of periodontal disease.

The mean $\mathrm{pS}$ and percentage of sites with detectable pS correlated well with the BANA test $(r=0.603, P$ $<0.001)$. This finding indicates that $\mathrm{pS}$ level is a possible predictor for three periodontal pathogens ( $T$. denticola, $P$. gingivalis, and $B$. forsythus) as well as for periodontal disease progression. However, $17.5 \%$ of sites that were BANA test negative showed detectable pS. There might be other microbes producing VSC in subgingival plaque as well. Efforts to clarify the association between $\mathrm{pS}$ and an expanded panel of subgingival bacteria are needed. Hence, pS level obtained using the sulfide monitor might provide adjunctive information for predicting the presence of periodontal pathogens in patients.

The sensitivity and specificity of pS measurements calculated by the $2 \times 2$ contingency table were $87 \%$ and $83 \%$, respectively, which were slightly higher than those of the BANA test (sensitivity, 84\%; specificity, 
$76 \%)$. This can be explained in part by the fact that VSC are produced by many periodontal pathogens, ${ }^{12}$ whereas the BANA test screens only for certain bacteria. ${ }^{13}$ Furthermore, VSC are potentially toxic compounds to the surrounding periodontal environment. ${ }^{1-6}$ Therefore, the direct measurement of pS levels might be more closely related to periodontal inflammation than the BANA test.

The concern of the BANA test is its reported low specificity. ${ }^{17,18}$ A cross-sectional study using 126 sites from 19 periodontal subjects showed that the BANA test had a sensitivity of $99 \%$, whereas it yielded only a specificity of $55 \% .{ }^{18}$ However, this study incubated the BANA test card at $55^{\circ} \mathrm{C}$ for 15 minutes. Studies have looked at different incubation times and temperatures based upon specificity. When a test card was incubated at $35^{\circ} \mathrm{C}$ for 5 minutes, the specificity increased to $68 \%{ }^{17}$ or $74 \% .{ }^{21}$ Our study yielded similar specificity of $76 \%$ using the same conditions.

Tests to screen for existing periodontal disease may utilize case-control and/or cross-sectional designs like this study. ${ }^{22}$ However, in order to confirm these observations, randomized, controlled, longitudinal studies are needed. ${ }^{22}$ It is also important to identify the status of periodontal disease (e.g., active or non-active) to enhance our ability to provide the most effective treatment. ${ }^{23}$ Results obtained from this study indicate that pS level may have potential to detect disease activity. However, more controlled studies are still needed to confirm this hypothesis.

In summary, sulfide levels in periodontal pockets increased significantly in sites with increased radiographic bone loss. The pS levels were positively correlated with the BANA test. It is then suggested that pS level may be a potential indicator for detecting the severity of periodontal disease.

\section{ACKNOWLEDGMENTS}

This study was supported in part by Diamond General Development Corp., Ann Arbor, Michigan.

\section{REFERENCES}

1. Ng W, Tonzetich J. Effect of hydrogen sulfide and methyl mercaptan on the permeability of oral mucosa. J Dent Res 1984;63:994-997.

2. Johnson PW, Yaegaki K, Tonzetich J. Effect of volatile thiol compounds on protein metabolism by human gingival fibroblasts. J Periodont Res 1992;27:553-561.

3. Johnson PW, Ng W, Tonzetich J. Modulation of human gingival fibroblast cell metabolism by methyl mercaptan. $J$ Periodont Res 1992;27:476-483.

4. Johnson P, Yaegaki K, Tonzetich J. Effect of methyl mercaptan on synthesis and degradation of collagen. J Periodont Res 1996;31:323-329.

5. Lancero H, Niu J, Johnson PW. Exposure of periodontal ligament cells to methyl mercaptan reduces intracellular $\mathrm{pH}$ and inhibits cell migration. $J$ Dent Res 1996;75:1994-2002.

6. Ratcliff PA, Johnson PW. The relationship between oral malodor, gingivitis, and periodontitis. A review. J Peri- odontol 1999;70:485-489.

7. Solis-Gaffar MC, Rustogi KN, Gaffar A. Hydrogen sulfide production from gingival crevicular fluid. $J$ Periodontol 1980;51:603-606.

8. Rizzo AA. The possible role of hydrogen sulfide in human periodontal disease. I. Hydrogen sulfide production in periodontal pockets. Periodontics 1967;5:233-236.

9. Coil JM, Tonzetich J. Characterization of volatile sulphur compound production at individual gingival crevicular sites in humans. J Clin Dent 1992;3:97-103.

10. Persson S. Hydrogen sulfide and methyl mercaptan in periodontal pockets. Oral Microbiol Immunol 1992;7: 378-379.

11. Persson S, Claesson R, Carlsson J. The capacity of subgingival microbiotas to produce volatile sulfur compounds in human serum. Oral Microbiol Immunol 1989; 4:169-172.

12. Persson S, Edlund MB, Claesson R, Carlsson J. The formation of hydrogen sulfide and methyl mercaptan by oral bacteria. Oral Microbiol Immunol 1990;5:195-201.

13. Loesche WJ, Bretz WA, Kerschensteiner D, et al. Development of a diagnostic test for anaerobic periodontal infections based on plaque hydrolysis of benzoyl-DLarginine-naphthylamide. J Clin Microbiol 1990;28:15511559.

14. Beck JD, Koch GG, Rozier RG, Tudor GE. Prevalence and risk indicators for periodontal attachment loss in a population of older community-dwelling blacks and whites. J Periodontol 1990;61:521-528.

15. Machtei EE, Hausmann E, Grossi SG, Dunford R, Genco RJ. The relationship between radiographic and clinical changes in the periodontium. J Periodont Res 1997;32: 661-666.

16. Grossi SG, Genco RJ, Machtei EE, et al. Assessment of risk for periodontal disease. II. Risk indicators for alveolar bone loss. J Periodontol 1995;66:23-29.

17. Loesche WJ, Kazor CE, Taylor GW. The optimization of the BANA test as a screening instrument for gingivitis among subjects seeking dental treatment. $J$ Clin Periodontol 1997;24:718-726.

18. Hemmings KW, Griffiths GS, Bulman JS. Detection of neutral protease (Periocheck) and BANA hydrolase (Perioscan) compared with traditional clinical methods of diagnosis and monitoring of chronic inflammatory periodontal disease. J Clin Periodontol 1997;24:110-114.

19. Fine DH, Mandel ID. Indicators of periodontal disease activity: An evaluation. J Clin Periodontol 1986;13:533-546.

20. Löe H, Silness J. Periodontal disease in pregnancy. I. Prevalence and severity. Acta Odontol Scand 1963;21: 533-551.

21. Feitosa ACR, Amalfitano J, Loesche WJ. The effect of incubation temperature on the specificity of the BANA (N-benzoyl-DL-arginine-naphthylamide) test. Oral Microbiol Immunol 1993;8:57-61.

22. Council on Scientific Affairs, American Dental Association. Products and methods for the diagnosis and/or management of periodontitis. J Periodontol 1998;69: 1071-1075.

23. Armitage GC. Periodontal diseases: Diagnosis. Ann Periodontol 1996;1:37-215.

Send reprint requests to: Dr. Hom-Lay Wang, Department of Periodontics/Prevention/Geriatrics, University of Michigan School of Dentistry, 1011 N. University Avenue, Ann Arbor, MI 48109-1078. Fax: 734/936-0374; e-mail: homlay@umich.edu

Accepted for publication July 7, 2000. 